“ (C) 2018 IEEE. Personal use of this material is permitted. Permission from IEEE must be obtained for all other uses, in any current or future media, including

reprinting/republishing this material for advertising or promotional purposes, creating new collective works, for resale or redistribution to servers or lists, or reuse of any copyrighted component of this work in other works." 


\section{Fuzzy Adaptive PI Decoupling Control for Gas Supply System of Proton Exchange Membrane Fuel Cell}

\author{
Jing Chen \\ Department of Automation \\ Kunming University of Science and \\ Technology \\ Kunming, China \\ 530614263@qq.com \\ Jianguo Zhu \\ School of Electrical \& Information \\ Engineering \\ University of Sydney \\ Sydney, Australia \\ jianguo.zhu@sydney.edu.au
}

\author{
Yuedong Zhan \\ Department of Automation \\ Kunming University of Science and \\ Technology \\ Kunming, China \\ ydzhan@163.com \\ $\mathrm{Li} \mathrm{Li}$ \\ Department of Automation \\ Kunming University of Science and \\ Technology \\ Kunming, China \\ kmustlili@163.com
}

\author{
Youguang Guo \\ School of Electrical and Data \\ Engineering \\ University of Technology Sydney \\ Sydney, Australia \\ youguang.guo-1@uts.edu.au \\ Bo Liang \\ School of Materials and Energy \\ Guangdong University of Technology \\ Guangzhou, China \\ liangbo@gdut.edu.cn
}

\begin{abstract}
Aiming at the issues of nonlinear and strong coupling between hydrogen partial pressure and oxygen partial pressure in a proton exchange membrane fuel cell (PEMFC) and the poor control effect of traditional PID method, a fuzzy adaptive PID decoupling control method with simple rules is proposed in this paper. A fuzzy control algorithm is used to realize the adaptive adjustment of PID parameters online and the decoupling of the PEMFC gas supply system. According to the model established, the PEMFC gas supply system with fuzzy adaptive PID decoupling control is simulated. Simulation results show that the proposed control strategy can effectively reduce the pressure difference between hydrogen and oxygen supplies, prevent the PEMFC from degradation, and improve the output performance and lifetime of PEMFC.
\end{abstract}

Keywords-Proton exchange membrane fuel cell (PEMFC); Hydrogen pressure; Oxygen pressure; Decoupling control; Fuzzy adaptive PID control

\section{INTRODUCTION}

The development of renewable energy is being impelled by the energy and environment crisis. Enhancing research and application of renewable energy has become an inevitable trend in the world. The fuel cell $(\mathrm{FC})$, as a high efficient and environment-friendly power generating device is a new high-tech, which has a great influence on human society in the 21 st century and has been paid great attention to from all over the world. As the fifth generation FC [1], the proton exchange membrane fuel cell (PEMFC) not only has the complementarity with the wind energy and solar energy, but also has the advantages of low working temperature, low working noise, low environment pollution, high efficiency, flexible use and so on [2]. In summary, PEMFC has a good prospect for the commercial development.

The PEMFC is a kind of power generating equipment, which converts chemical energy into electric energy directly through electrochemical reaction by using hydrogen as fuel and oxygen as oxidant. Because the PEMFC gas supply system is of nonlinear and strongly-coupling, there are plenty of academics who have looked into the researches in this field. As for the decoupling control of PEMFC gas supply system, Yan [3] proposed a fuzzy control algorithm for the nonlinearity of the temperature and flow control system of the reformer plant in a solid oxide fuel cell (SOFC). Simulation results showed that the control algorithm mitigated the coupling between variables. In view of the nonlinear and strong coupling of the air flow and pressure of the fuel cell, Wei [4] used a recursive identification algorithm to identify the model parameters of the control channel and the coupling channel of the control variables online, and designed the decoupling matrix. Then, the decoupling control of the air flow and pressure is realized by adjusting the parameters of the decoupling matrix in real time. Ly et al. [5] adjusted the humidity of fuel cell cathode by single neuron PID control strategy with quadratic performance index and controlled the air flow by incremental PID control algorithm based on dynamic identification of diagonal regression neural network. The hybrid intelligent PID decoupling controller overcomes the nonlinearity and coupling of the system, and reduces the bad influence on the real-time power demand of the load. Yang et al. [6] proposed a bivariate dynamic decoupling PID control strategy based on the quasi diagonal recurrent neural network, which decoupled the temperature system of the gas turbine hybrid power generation system based on molten carbonate fuel cell, so that the components of the system can be operated in ideal temperature range. Zhao et al. [7] proposed a dynamic disturbance decoupling strategy based on the active disturbance rejection control to adjust the mass flow and gas pressure of fuel cell. Experiments show that the proposed method has better output performance than the traditional PID method in cases of transient and steady state. Chen et al. [8] realized the decoupling control of air flow and back pressure in the air supply system of fuel cell by identifying the transfer function of the system, so that the system had a good robustness at load changing. Chen et al. [9] presented an improved temperature control method to improve the control accuracy and the response performance of temperature of inlet cooling water in view of the temperature rising when current surges. Li et al. [10] established an analytical model which greatly reduces the numerical complexity of the multivariable system models. Na and Gou [11] proposed a nonlinear control strategy to decouple the gas supply system completely, and reduce the partial pressure difference between hydrogen and oxygen effectively.

This work was supported in part by the National Natural Science Foundation of China under Grant 51667012 


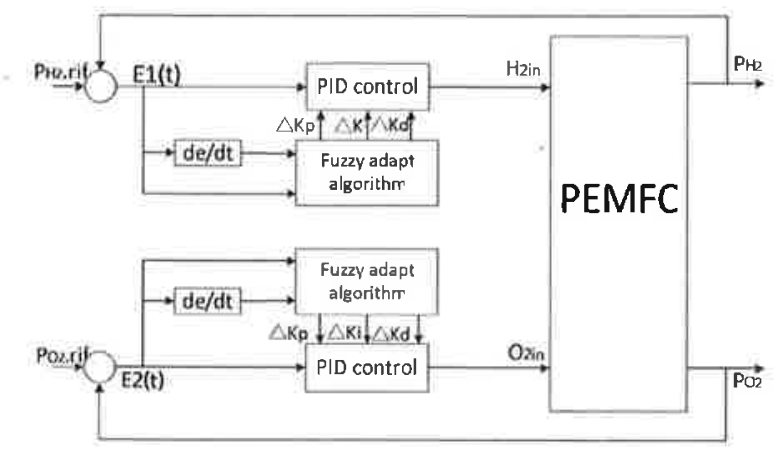

Fig. 1. Structure diagram of the proposed fuzzy adaptive PID decoupling controller.

However, the above control methods generally require complex mathematical operations and cannot adapt to the response of the system dynamically. Due to the intricate working environment and load changes of $\mathrm{FC}$, the intelligent adaptive decoupling control algorithms need to be applied to FC control systems. In this paper, a fuzzy adaptive PID decoupling controller is formed by combining an improved fuzzy adaptive algorithm with PID control algorithm on PEMFC hydrogen partial pressure loop and oxygen partial pressure loop, respectively. Fuzzy adaptive algorithm is added to PID controller to form a fuzzy-PID adaptive decoupling controller. The proposed control algorithm can realize the dynamic decoupling compensation by decoupling the coupling loops into two single loops. The block diagram of the fuzzy adaptive PID decoupling system is shown in Fig. 1.

This paper conducts the study on the fuzzy adaptive PID decoupling control for the PEMFC gas supply system and the fuzzy adaptive PID decoupling control is simulated. The rest of this paper is organized in the following. Section 2 presents the PEMFC gas supply system model. Section 3 analyzes the traditional PID control performance of PEMFC gas supply system and designs the fuzzy adaptive PID decoupling controller. In Section 4, the simulation results are presented to show that the proposed control strategy can effectively reduce the pressure difference between hydrogen and oxygen supplies

\section{PRESSURE DifFERENCE MODELS OF PEMFC BETWEEN ITS ANODE AND CATHODE}

Take the $P_{\mathrm{H}_{2}}$ and $P_{\mathrm{H}_{2} \mathrm{O}}$ in anode and $P_{\mathrm{O}_{2}} 、 P_{\mathrm{N}_{2}}$ and $P_{\mathrm{H}_{2} \mathrm{O}_{\mathrm{c}}}$ in cathode of PEMFC as the state variables. According to the law of molar conservation, the rate of velocity changes of $\mathrm{H}_{2}$ and $\mathrm{O}_{2}$ is equal to the inlet velocity minus the outlet velocity and the rate of electrochemical reaction of $\mathrm{H}_{2}$ and $\mathrm{O}_{2}$; the rate of velocity change of $\mathrm{N}_{2}$ can be calculated from the import rate minus the export rate of $\mathrm{N}_{2} \cdot \mathrm{H}_{2} \mathrm{O}$ is the electrochemical reaction product of PEMFC and penetrated through the proton exchange membrane between the cathode and anode. Therefore, the rate of changes of $P_{\mathrm{H}_{2} \mathrm{O}_{\mathrm{s}}}$ and $P_{\mathrm{H}_{2} \mathrm{O}}$ can be calculated from the sum of the velocity of the steam inlet flow in anode and cathode and generation rate of steam in the electrochemical reaction minus the outlet velocity, when considering the transfer through the proton membrane between the cathode and the anode. Finally, the state equation of PEMFC gas supply system can be obtained through detailed analysis and combination with the ideal gas theorem on the above basis.

The PEMFC gas supply system can be described as:

$$
\begin{aligned}
& \frac{\mathrm{d} P_{m H_{2}}}{\mathrm{~d} t}=\frac{R T}{V_{t 1}}\left[K K_{1} H_{2 m}-C_{1} I_{f c}-\left(K_{1} H_{2 m}-C_{1} I_{f c}\right) F_{t I_{2}}\right] \\
& \frac{\mathrm{d} P_{H_{2} O_{a}}}{\mathrm{~d} t}=\frac{R T}{V_{A}}\left[K H_{2 l m}\left(\frac{\varphi_{a} P_{V S}}{P_{H_{2}}+P_{H_{2} \mathrm{Oa}_{a}}-\varphi_{a} P_{V S}}\right)\right. \\
& \left.-\left(K_{1} H_{2 m}-C_{1} I_{f c}\right) F_{H_{2} O_{a}}-C_{2} I_{f c}\right] \\
& \frac{\mathrm{d} P_{O_{2}}}{\mathrm{~d} t}=\frac{R T}{V_{C}}\left[K K_{2} O_{2 i n}-\frac{C_{2}}{2} I_{f c}-\left(K_{2} O_{2 i n}-\frac{C_{2}}{2} I_{f c}\right) F_{O_{2}}\right] \\
& \frac{\mathrm{d} P_{N_{2}}}{\mathrm{~d} t}=\frac{R T}{V_{C}}\left[K K_{2} O_{2 i n}-K_{2} O_{2 i n} F_{N_{2}}\right] \\
& \frac{\mathrm{d} P_{H, O_{c}}}{\mathrm{~d} t}=\frac{R T}{V_{C}}\left[K_{2} O_{m 1} \frac{\varphi_{c} P_{V S}}{P_{O_{2}}+P_{N_{2}}+P_{l l_{2} O_{c}}-\varphi_{c} P_{V S}}+\left(C_{1}+C_{2}\right) I_{f c}\right. \\
& \left.-\left(K_{2} O_{m+1}+C_{1} I_{f c}+C_{2} I_{f c}\right) F_{l l, O} O_{\mathrm{r}}\right]
\end{aligned}
$$

The actual output voltage of PEMFC stack can be expressed as:

$$
\begin{aligned}
V_{\text {slack }}=N & {\left[E^{\prime \prime}+\frac{R T}{2 F} \ln \left(\frac{P_{t l_{i} \sqrt{P_{O_{i}}}}}{P_{t l_{2} O_{s}}}\right)-\frac{R T}{2 \alpha F} \ln \left(A_{f_{c}} I_{f c}\right)\right.} \\
& \left.-R I_{f c}-m \exp \left(n I_{f c}\right)\right]
\end{aligned}
$$

where $F_{\mathrm{O}_{2}}=P_{\mathrm{O}_{2}} / P_{\mathrm{O}_{2}}+P_{N_{2}}+P_{\mathrm{H}_{2} \mathrm{O}_{\mathrm{c}}}, F_{\mathrm{N}_{2}}=P_{\mathrm{N}_{2}} / P_{\mathrm{O}_{1}}+P_{\mathrm{N}_{2}}+P_{I I_{2} \mathrm{O}_{\mathrm{c}}}$, $F_{\mathrm{H}_{2} \mathrm{O}_{\mathrm{r}}}=P_{\mathrm{H}_{2} \mathrm{O}_{\mathrm{c}}} / P_{\mathrm{O}_{2}}+P_{\mathrm{N}_{2}}+P_{\mathrm{H}_{2} \mathrm{O}_{\mathrm{c}}} \quad, \quad F_{\mathrm{H}_{2}}=P_{\mathrm{H}_{2}} / P_{\mathrm{H}_{2}}+P_{\mathrm{H}_{2} \mathrm{O}_{\mathrm{a}}}$, $F_{\mathrm{H}_{2} \mathrm{O}_{a}}=P_{\mathrm{H}_{2} \mathrm{O}_{a}} / P_{\mathrm{H}_{2}}+P_{\mathrm{H}_{2} \mathrm{O}_{a}}, P_{\mathrm{H}_{2}}, P_{\mathrm{O}_{2}} P_{\mathrm{N}_{2}} P_{\mathrm{H}_{2} \mathrm{O}_{a}} P_{\mathrm{H}_{2} \mathrm{O}_{c}}$ are the pressures of the hydrogen, oxygen, nitrogen, anode steam, and cathode steam, respectively $(k P a), P$ the gas pressure $(k P a), V$ the gas volume $\left(\mathrm{cm}^{3}\right), n$ the amount of gas substance $(\mathrm{mol}), R$ the gas constant $(8.314 \mathrm{~J} /(\mathrm{mol} \cdot \mathrm{K})), T$ the temperature $(K), v_{A}, v_{C}$ the volume of anode and cathode, respectively $\left(\mathrm{m}^{3}\right), N$ the number of fuel cells, $H_{2, m}, O_{2, m}$ the gas flow velocity $(\mathrm{mol} / \mathrm{s}), F$ the Faraday constant $(96485 \mathrm{C} / \mathrm{mol}), A_{j c}$ the effective fuel cell area $\left(\mathrm{m}^{2}\right)$, $I_{f c}$ the fuel cell output current $(A), P_{v s}$ the gas saturation pressure at the temperature $353 \mathrm{~K}(\mathrm{KPa}), E^{0}$ the open circuit voltage of PEMFC under standard pressure $(V), \alpha$ the charge transfer coefficient, and $n, m$ the mass transfer voltage constants.

In a PEMFC power generation system (PGS), the pressure and flow of the reaction gas in the PEMFC are to change when the load changes. In addition, the output voltage of the PEMFC is a function of the gas pressure mentioned above, and the gas pressure has greater influence 
on the performance of the $\mathrm{FC}$ than the other parameters [12]. Meanwhile, it is difficult to control the gas supplies of PEMFC because of its strong coupling properties and nonlinearity [13]. Hence, an effective decoupling control method is necessary for PEMFC PGS to prevent the damage of PEMFC and improve its output performance.

\section{DESIGN OF FUZZY ADAPTIVE PID DECOUPLING CONTROLLER}

\section{A. Traditional PID controller}

Because of its simple structure, strong handling ability and great robustness, the traditional PI controller is still widely used in industrial process control. The standard form of PI control can be written as follows.

$$
\begin{gathered}
u(t)=K_{p} e(t)+K_{i} \int_{0}^{t} e(t) d t+K_{d} \frac{d e(t)}{d t} \\
e(t)=y(t)-r(t)
\end{gathered}
$$

where $e$ is the error between the actual value $y$ and the reference value $r, u$ the control signal, $K_{p}$ the proportional gain regulating system accuracy, $K_{i}$ the integral gain eliminating the steady state error, and $K_{d}$ the derivative gain improving the dynamic characteristics of the system.

Based on the established PEMFC gas state equation above, $H_{2 \text { in }}$ and $O_{2 \text { in }}$ are used as the control variables, $P_{H_{2}}$ and $P_{O_{2}}$ are the output variables, and $I_{f c}$ is the disturbance variable. The PI controller of PEMFC stack is constructed by using the Matlab/Simulink, as shown in Figs. 2 and 3.

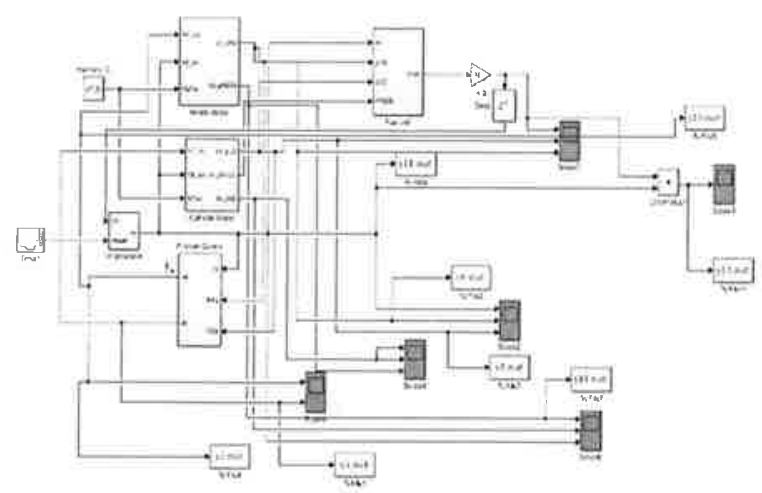

Fig. 2. Matlab/Simulink structure diagram for PEMFC control system

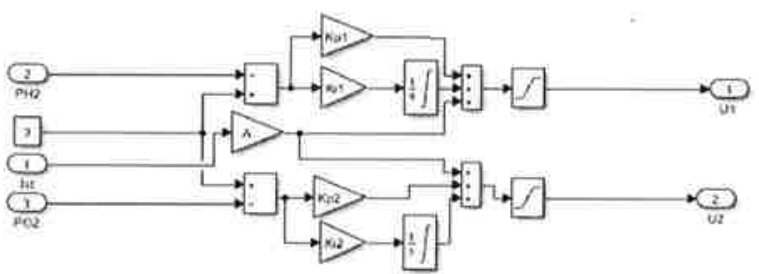

Fig. 3. PI control system

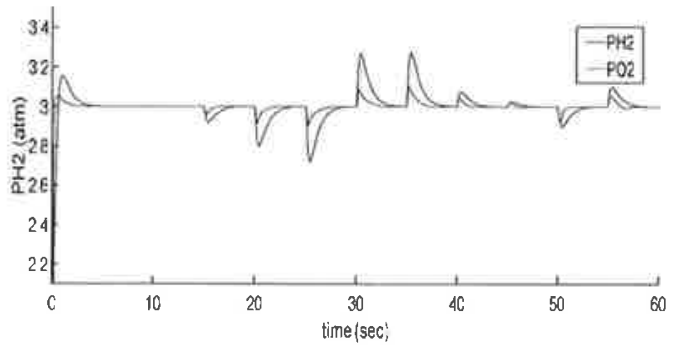

Fig 4. Pressure waveforms with load changing and transitional PI control.

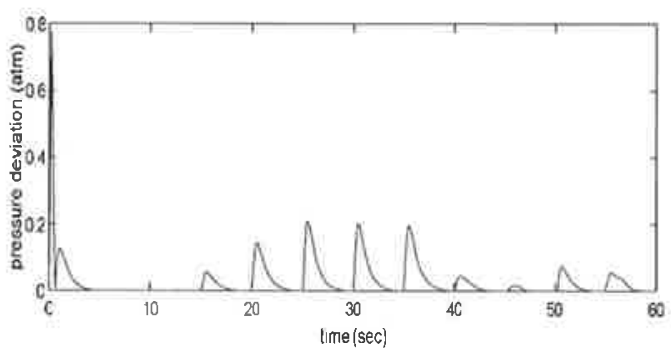

Fig 5 Performance curve of pressure difference between $P_{\mathrm{H}_{2}}$ and $P_{\mathrm{O}_{2}}$ with traditional PI control

Set the PI parameters with the error experiment method, $K_{p \mid 1}=5, K_{\mathrm{i} 1}=5$, or $K_{P 2}=50, K_{\mathrm{i} 2}=50$. The simulation results are shown in Figs. 4 and 5.

From Figs. 4 and 5, it can be seen that there is a poor control performances of pressure difference between $P_{H_{2}}$ and $P_{\mathrm{O}_{2}}$.

\section{B. Fuzzy Adaptive PID Decoupling Controller}

In this paper, the fuzzy adaptive PID decoupling controller proposed is the improvement of the traditional PID controller, which adjusts parameters of PID controller automatically. The fuzzy adaptive PID decoupling controller takes the error and the error rate as the input variables, and adjusts the parameters of the PID controller according to the fuzzy rules in order to meet the requirements of the selftuning PID controller. The design process of the fuzzy controller consists of three main steps: fuzzification, fuzzy inference and deblurring.

The input variables are $e$ and $e c$, and the output variables are $\Delta K_{p} 、 \Delta K_{i}$ and $\Delta K_{d}$, which can be given by:

$$
\left.\begin{array}{c}
e=r-y \\
e c=d e / d t \\
K_{p}=K_{p 0}+\Delta K_{p} \\
K_{i}=K_{i 0}+\Delta K_{i} \\
K_{d}=K_{d 0}+\Delta K_{d}
\end{array}\right\}
$$

where $e$ is the difference between the actual output $y$ and the reference $r, e c$ the variation ratio of $e, K_{p 0}, K_{i 0}$, and $K_{d 0}$ are the initial parameters of PID controller, and $K_{p}, K_{i}$, and $K_{d}$ the parameters of PID controller adjusted by fuzzy adaptive algorithm online, respectively. 
The triangle membership function is adapted because of briefness and occupying less memory, which can be describes as:

$$
\mu(x)=\left\{\begin{array}{l}
\frac{x-a}{b-a}, x \in(a, b) \\
\frac{x-c}{b-c}, x \in(b, c)
\end{array}\right.
$$

where $a, b, c$ are the constants of fuzzy domain, respectively.

According to the response curve and experiments, the universals of $e, e c, \Delta K_{p}, \Delta K_{i}$ in hydrogen flow loop are set as $[0,0.11],[-1,1],[-6,6],[-4,4]$, respectively. The membership functions of $e$ are depicted in Figs. 6 and 7. In oxygen flow loop, the universals of $e, e c, \Delta K_{p}, \Delta K_{i}$ in hydrogen flow loop are set as $[0,0.09],[-1,1],[-15,15][-$ $10,10]$.

The core of the fuzzy controller design is the formulation of fuzzy rules. According to the influence of parameters on the control system, i.e., the increasing of proportion parameters will cause the overshoot enlarging and the system response time decreasing; with the increase of integral parameters, the overshoot and callback ratio will increase while the stability of the system will be weakened. To sum up, the rules of fuzzy inference is formulated based on the actual output of PEMFC model, which can be described as Tables I and II, respectively.

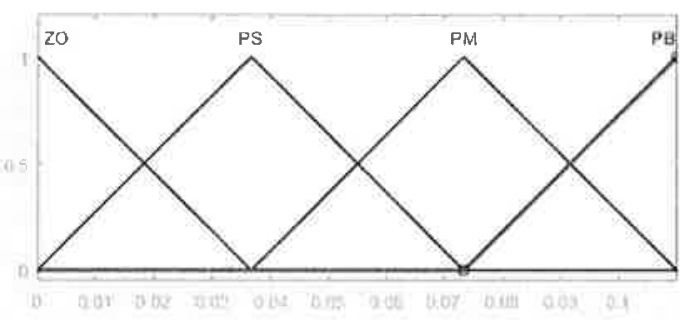

Fig. 6. The membership function of $e$ of $P_{H_{2}}$.

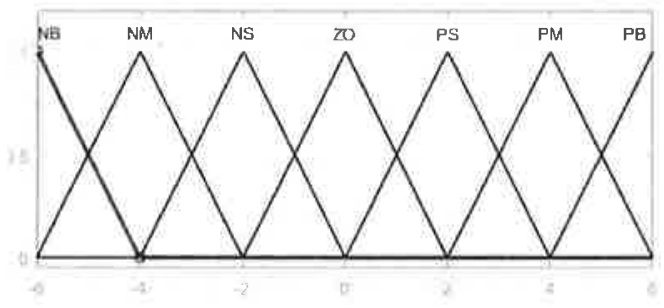

Fig. 7. The membership function of $\Delta K_{p}$ of $P_{H_{2}}$.
TABLE I $\quad$ FUZzY RULES OF Proportional GaIN $\Delta K_{p}$

\begin{tabular}{|c|c|c|c|c|c|}
\hline \multicolumn{2}{|c|}{$\Delta K_{p}$} & \multicolumn{4}{|c|}{$e$} \\
\cline { 2 - 6 } & ZO & PS & PM & PB \\
\hline \multirow{4}{*}{ ec } & NB & ZO & ZO & ZO & PS \\
\cline { 2 - 6 } & NM & ZO & ZO & PS & PS \\
\cline { 2 - 6 } & NS & PS & PS & PS & PM \\
\cline { 2 - 6 } & ZO & PS & PS & PM & PM \\
\cline { 2 - 6 } & PS & PS & PM & PM & PB \\
\cline { 2 - 6 } & PM & PM & PM & PB & PB \\
\cline { 2 - 6 } & PB & PM & PB & PB & PB \\
\hline
\end{tabular}

TABLE IL FUzzY RULES OF INTEGRAL GAIN $\Delta K_{i}$.

\begin{tabular}{|c|c|c|c|c|c|}
\hline \multirow{2}{*}{$\Delta K_{i}$} & \multicolumn{4}{|c|}{$e$} \\
\cline { 2 - 6 } & ZO & PS & PM & PB \\
\hline \multirow{4}{*}{ ec } & NB & NM & NM & ZO & ZO \\
\cline { 2 - 6 } & NM & NM & NS & ZO & ZO \\
\cline { 2 - 6 } & NS & NS & ZO & PS & PS \\
\cline { 2 - 6 } & ZO & ZO & PS & PS & PM \\
\cline { 2 - 6 } & PS & PS & PS & PM & PM \\
\cline { 2 - 6 } & PM & PM & PM & PB & PB \\
\cline { 2 - 6 } & PB & PM & PB & PB & PB \\
\hline
\end{tabular}

The weighted average method in ambiguity resolution can be described as:

$$
Z_{0}=\frac{\sum_{i=1}^{n} Z_{i} \mu_{c}\left(Z_{i}\right)}{\sum_{i=1}^{n} \mu_{c}\left(Z_{i}\right)}
$$

where $Z_{0}$ is the clear value, $Z_{1}$ the membership value, and $\mu_{c}\left(Z_{i}\right)$ the fuzzy variable.

\section{EXPERIMENTAL SETUP AND RESULTS}

The proposed fuzzy adaptive PID decoupling control is tested by using MATLAB/Simulink module, and the structure of the control system for $P_{H_{2}}$ is shown in Fig. 8.

The parameters of the PEMFC model are listed Table III and the parameters of the PID controller are adjusted off-line. The simulation results show that the whole system will achieve the best control effect when the parameters of PID controller are $K_{p}=15, K_{i}=10$ and $K_{d}=0$ in hydrogen loop and $K_{p}=65, K_{i}=50$ and $K_{d}=0$ in oxygen loop. 


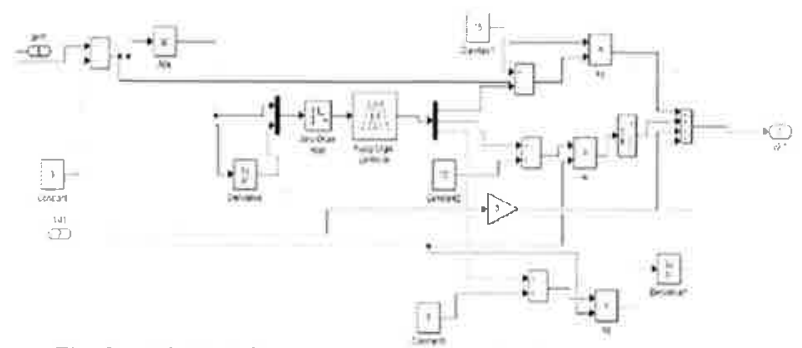

Fig. 8. Adaptive fuzzy self-tuning PID decoupling control system.

TABLE III PEMFC SIMULATION PARAMETERS.

\begin{tabular}{|c|c|c|}
\hline Definition & Parameter & Value \\
\hline Cell number & $N$ & 35 \\
\hline Open-circuit cell voltage & $E_{0}$ & $1.032 \mathrm{~V}$ \\
\hline Universal gas constant & $R$ & $\begin{array}{c}8314 \\
\mathrm{~J} /(\mathrm{mol} \cdot \mathrm{K})\end{array}$ \\
\hline Faraday constant & $F$ & $\begin{array}{l}96485 \\
\mathrm{C} / \mathrm{mol} \\
\end{array}$ \\
\hline Charge transfer coefficient & $\alpha$ & 0.5 \\
\hline PEMFC active area & $A_{f c}$ & $232 \mathrm{~cm}^{2}$ \\
\hline Anode volume & $V_{a}$ & $0,005 \mathrm{~m}^{3}$ \\
\hline Cathode volume & $V_{c}$ & $001 \mathrm{~m}^{3}$ \\
\hline PEMFC working temperature & $T$ & $338.5 \mathrm{~K}$ \\
\hline $\begin{array}{c}\text { Constant in the mass transfer } \\
\text { voltage }\end{array}$ & $m$ & $\begin{array}{c}2.11 \times 10^{-5} \\
\mathrm{~V}\end{array}$ \\
\hline $\begin{array}{c}\text { Constant in the mass transfer } \\
\text { voltage }\end{array}$ & $n$ & $\begin{array}{c}8 \times 10^{3} \\
\mathrm{~cm}^{2} \mathrm{~mA}^{2}\end{array}$ \\
\hline Area resistance & $r$ & $\begin{array}{c}245 \\
\Omega \cdot \mathrm{cm}^{2}\end{array}$ \\
\hline Saturated gas pressure at $335 \mathrm{~K}$ & $P_{v s}$ & $32 \mathrm{kPa}$ \\
\hline Anode conversion factor & $k_{a}$ & $\begin{array}{c}7.034 \times \\
10^{-4} \mathrm{~mol} / \mathrm{s}\end{array}$ \\
\hline Cathode conversion factor & $k_{c}$ & $\begin{array}{c}7.036 \times \\
10^{-4} \mathrm{~mol} / \mathrm{s}\end{array}$ \\
\hline
\end{tabular}

The aim of the proposed control is to keep the partial pressure of $\mathrm{H}_{2}$ and $\mathrm{O}_{2}$ at $3 \mathrm{~atm}(1 \mathrm{~atm}=0.1 \mathrm{MPa})$ at load changing, and minimize the pressure difference between pressures of $\mathrm{H}_{2}$ and $\mathrm{O}_{2}$. The transitional waveform of load changing can be described as Fig. 9.

By comparing the simulation results of two methods as shown in Fig. 10, it is concluded that the proposed control strategy can not only reduce the difference value of PEMFC's cathode and anode by $25 \%$, but also realize the smooth static tracking for set pressure. The output voltage between the fuzzy adaptive PID decoupling and the traditional PID is not obvious, because the response time is less than a few milliseconds according to literature, as depicted in Fig. 11.

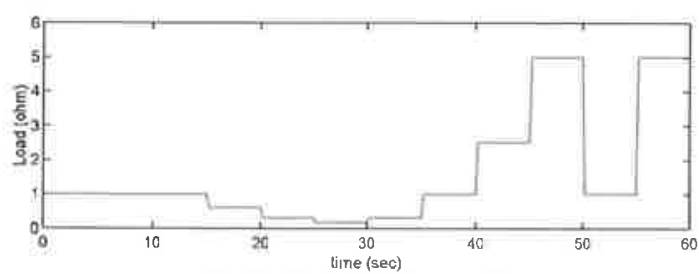

Fig. 9. Load variation profile.

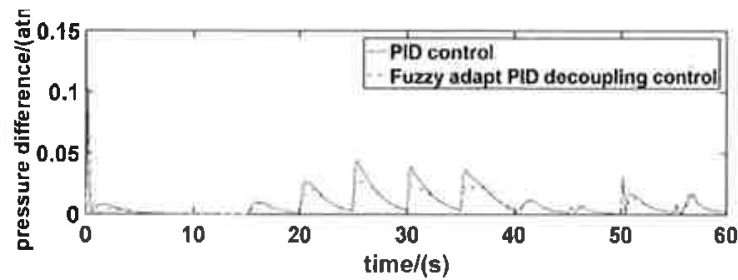

Fig. 10. Performance curve of pressure difference between two control strategies.

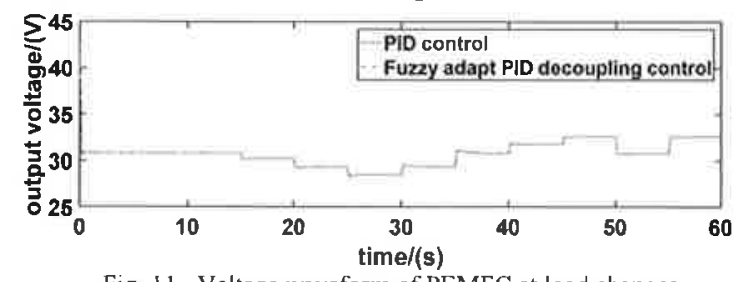

Fig i1 Voltage waveform of PEMFC at load changes.

\section{CONCLUSION}

Based on the overview of the decoupling control technology of the PEMFC gas supply system, a fuzzy adaptive PID decoupling control is presented to realize the decoupling control of the PEMFC gas supply system. By comparing the simulation results with traditional PID control, the proposed control strategy not only reduces the algorithm complexity, but also improves the robustness of system, and reduces the difference pressure between hydrogen and oxygen efficiently.

\section{REFERENCES}

[1] J. Larminie, and A Dicks. Fuel Cell Systems Explained, 2nd ed. Chichester: John Wiley \& Sons Ltd, 2003.

[2] M. V. Lototskyy, et al., "Metal hydride hydrogen storage and supply systems for electric forklift with low-temperature proton exchange membrane fuel cell power module," Int. J. Hydrogen Energy, vol, 41, no, 31, pp 13831-13842, 2016

[3] H Yan, "Modeling of SOFC reformer thermal management and decoupling control strategy," Wuhan: Huazhong University of Science and Technology, 2009

[4] G. A. Wei, "Modeling and control strategy of air supply system in PEMFC in electric vehicle," Wuhan: Wuhan University of Technology, 2010

[5] H. Ly, E. Birgersson, and M, Vynnycky, "Computationally etficient multi-phase models for a proton exchange membrane fuel cell Asymptotic reduction and thermal decoupling," Int I Hydrogen Energy, vol. 36, no. 22, pp. 14573-14589, 2011.

[6] Y. Fan, and L Hao, "Temperature decoupling control for molten carbonate fuel cell/gas tubine hybrid power generation system," J Shanghai University of Electric Power, vol. 28, no. 4, pp 309-3/2, 2012

[7] D. Zhao, B. Blunier, F. Gao, M. Dou, and A Miraoui, "Disturbance decoupling control of an ultra-high speed centrifugal compressor for the air management of fuel cell systems," International Journal of Hydrogen Energy; vol 39, no 4, pp. 1788-1798, 2014

[8] F. Chen, J Chen, S. Xu, and T. Zhang, "Decoupling controller design for air supply subsystem of high pressure PEMFC system," J Tongji University (Natural Science), vol, 42, no 7, pp. 1096-1100, 2014.

[9] W Chen, Y Li, and X Zhao, "Temperature control strategy for water-cooled proton exchange membrane fuel cells," J Southwest Jiaotong University, vol. 50, no. 3, pp.393-399, 2015.

[10] L. Ang and L Zijing, "Efficient Inass transport and electrochemistry coupling scheme for reliable multiphysics modeling of planar solid oxide fuel cell stack," Chinese J. Chemical Pliysics, vol. 30, no. 2, pp 139-146, 2017

[1I] W. K Na and B. Gou, "Feedlback-Linearization-Based Nonlinear Control for PEM Fuel Cells," IEEE Trans Energy Conversion, vol. 23, no. I, pp 179-190, 2008 
[12] M. Movahedi, A Ramiar, and A A Ranjber, "3D numerical investigation of PID clamng pressure effect on the performance of proton exchange membrane fuel cell with Interdigitated Flow Field," Energy, in Press, 2018.
[13] E J Asensio, et al., "Model for optimal management of the cooling system of a fuel cell-based combined heat and power system for DeveloPIDng optimization control strategies," Applied Energy, vol 211 , no 5 , pp. $413-430,2018$. 